\title{
Perioperative Outcomes of Anatomic Endoscopic Enucleation of the Prostate, Robotic and Open Simple Prostatectomy From a Multi-Institutional Database
}

\author{
Muhieddine Labban, ${ }^{1}$ Nassib Abou Heidar, ${ }^{1}$ Vincent Misrai, ${ }^{2}$ Jad Najdi, ${ }^{1}$ Hani Tamim, ${ }^{3}$ Albert El-Hajj ${ }^{凶 1}$ \\ ${ }^{1}$ American University of Beirut Medical Center, Department of Surgery, Division of Urology, Beirut, Lebanon ${ }^{2}$ Department of Urology, Clinique Pasteur, Toulouse, France \\ ${ }^{3}$ American University of Beirut Medical Center, Clinical Research Institute, Beirut, Lebanon \\ $\mathrm{ML} \& \mathrm{NAH}$ contributed equally to the manuscript.
}

\section{Abstract}

Objective To compare the perioperative morbidity of robotic-assisted simple prostatectomy (RASP), anatomical endoscopic enucleation of the prostate (AEEP) and open simple prostatectomy (OSP) for the treatment of benign prostatic obstruction (BPO).

Methods The national surgical quality improvement program database was queried for AEEP, RASP, and OSP procedures. Clavien-Dindo-graded complications, length of hospital stay (LOS), and operative time were compared among the procedures. To control for the potentially confounding variables, we first conducted a multivariate backward conditional logistic regression, and then resorted to propensity score matching.

Results We identified 2867 AEEP, 234 RASP, and 1492 OSP procedures. After matching, the risk of pulmonary, renal, infectious, and thromboembolic adverse events was lower after AEEP but not RASP in comparison with OSP $(P<0.05)$. In comparison with RASP, AEEP had lower cardiac and thromboembolic events $(P<0.05)$. When compared with OSP, AEEP had reduced odds of Clavien-Dindo grade I (OR $=0.12 ; 95 \%$ CI 0.10 to 0.16$)$ and II $(\mathrm{OR}=0.38 ; 95 \%$ CI 0.24 to 9.58) complications. Also, AEEP had lower odds for grade I and II as well as grade IV complications $(\mathrm{OR}=$ $0.30 ; 95 \%$ CI 0.19 to 0.48 , and OR $=0.05$; $95 \%$ CI 0.01 to 0.24 , respectively) compared with RASP.

Conclusion AEEP and RASP were associated with fewer perioperative adverse events, a shorter LOS and a reduced risk of transfusion compared with OSP. AEEP was associated with overall lower complication rates than RASP and OSP.

\section{Introduction}

Benign prostatic obstruction (BPO) is a common cause of lower urinary tract symptoms with a $75 \%$ prevalence in men older than 50 years[1]. BPO significantly impacts quality of life, often necessitating medical or surgical treatment[2]. The current standard of care advocates for anatomical endoscopic enucleation of the prostate (AEEP) of smaller prostates $(<80$ grams) and recommends AEEP or simple prostatectomy for the treatment of larger prostates ( $>80$ grams) [3]. Nevertheless, open simple prostatectomy (OSP) remains the goldstandard treatment for large glands[4]. Recently, robotic-assisted simple prostatectomy (RASP) has gained popularity among robotic surgeons as a minimally invasive method to enucleate large prostatic adenomas.

\section{Key Words}

Benign prostatic obstruction, endoscopic enucleation of the prostate, perioperative outcomes, robotic-assisted simple prostatectomy

\section{Competing Interests}

$$
\text { None declared. }
$$

\section{Article Information}

Received on January 31, 2021

Accepted on March 27, 2021

Soc Int Urol J.2021;2(4):196-209

DOI: 10.48083/LKVV8843 


\section{Abbreviations \\ AEEP anatomical endoscopic enucleation of the prostate BPO benign prostatic obstruction \\ COPD chronic obstructive pulmonary disease \\ CPT current procedural terminology \\ HoLEP Holium laser enucleation of the prostate \\ LOS length of stay \\ MACE major adverse cardiac event \\ OSP open simple prostatectomy \\ RASP robotic-assisted simple prostatectomy}

It has similar functional outcome to OSP, while potentially limiting postoperative morbidity, especially bleeding and length of hospital stay [5-7].

The AEEP imitates the traditional Millins procedure by endoscopically enucleating the prostatic adenoma. Holium laser enucleation of the prostate (HoLEP) was first described by Gilling in 1998. In comparison to OSP, AEEP has demonstrated similar functional outcomes and efficacy in larger prostates, but reduced rate of overall complications and postoperative transfusion [8-10]. Currently, AEEP, RASP, and OSP are part of the surgical armamentarium when dealing with large prostates, and proponents of each technique defend its superiority over the others; however, there are no large studies comparing the 3 procedures, and certainly no randomized clinical trials. Umari et al. showed that RASP has comparable functional outcomes to AEEP, while Zhang et al. showed that AEEP conferred a shorter hospital stay, reduced catheter time, and had lower transfusion rates than RASP[11-13]. Both studies showed similar complication rates between RASP and AEEP. Nevertheless, both studies are small retrospective series. Sorokin et al. and Mourmouris et al. retrospectively compared RASP and OSP and found that despite having comparable functional outcomes, RASP had reduced blood loss and catheterization time at the expense of a longer operative time[7,14]. The systematic review of RASP by Kordan et al. incorporated several single-center retrospective studies, which included a limited number of RASP procedures[15]. Therefore, using a large multi-institutional real-world dataset, we sought to compare head-to-head the perioperative surgical outcomes of AEEP, RASP, and OSP using different statistical methods to cross-validate our findings.

\section{Materials and Methods}

The American College of Surgeons National Surgical Quality Improvement Program is a prospectively filled, risk-adjusted, and validated 30-day outcome registry with contributions from 703 different hospitals, most of which are in the United States[16]. Since our institution is a participating hospital, access to the deidentified database is granted and formal consent is not required. The database includes information on patients' demographics, comorbidities, and 30-day postoperative outcomes that are entered by American College of Surgeons (ACS) trained surgical clinical reviewers. The database was queried for AEEP, RASP, and OSP without any concurrent procedures performed between 2008 and 2017. Since there is no dedicated current procedural terminology (CPT) code for RASP, the CPT code for

\section{FIGURE 1.}

Flowchart describing the selection criteria for patients who underwent anatomic endoscopic enucleation of the prostate (AEEP) or robotic-assisted simple prostatectomy (RASP) or open simple prostatectomy (OSP) from the national surgical quality improvement program (NSQIP)

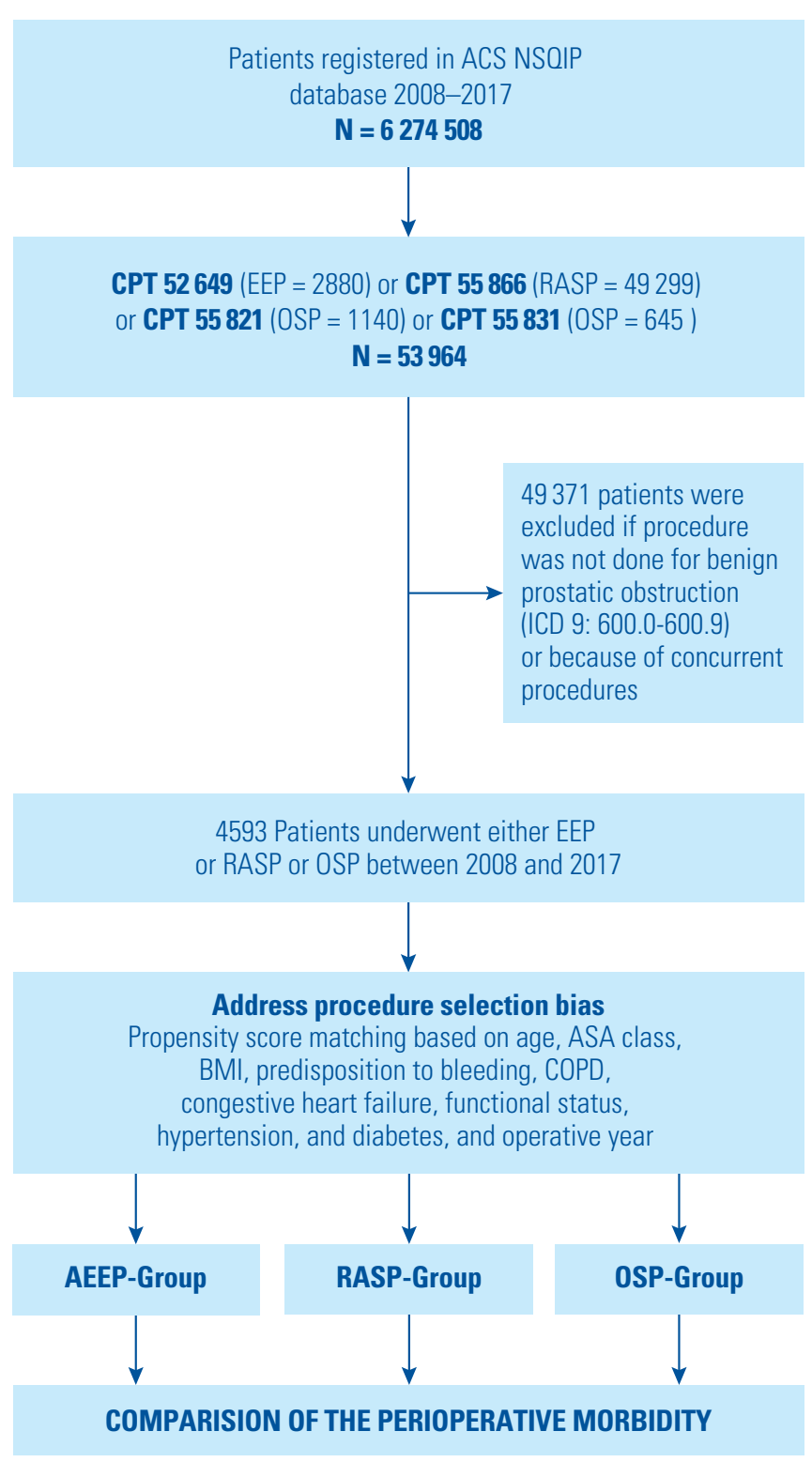


RARP (55866) is used by surgeons. To select procedures done for BPO, all cases were filtered by the International Classification of Diseases -9 (ICD-9) codes 600.00600.90 or ICD-10 N40.00-N40.90, corresponding to the postoperative diagnosis of BPO procured from either brief postoperative note, operative report, and/or the pathology reports (Figure 1). The CPT codes for AEEP (52649) and OSP (55821 \& 55831) were also selected. Even though the energy source for AEEP cannot be extracted from the database, HoLEP is currently the most commonly used energy source for AEEP in the United States[17]. As a primary endpoint, this study aimed to compare 30-day perioperative surgical complications and early mortality between AEEP, RASP, and OSP.

Baseline patient demographics, comorbidities, and laboratory markers were collected and managed. Comorbidities included American Society of Anesthesiologists (ASA) class $\geq 3$, corresponding to patients with severe systemic disease, diabetes, hypertension, being partially or totally physically dependent, chronic obstructive pulmonary disease (COPD), history of heart failure, myocardial infarction, and disseminated cancer. Additionally, the variables bleeding diathesis and long-term pharmaceutical steroid or immunosuppressant use were added. The variable bleeding diathesis incorporated patients on chronic anticoagulation therapy that had not been discontinued prior to the procedure, and patients with genetic hematological diseases. The variable chronic steroid use included patients who had regular intake of oral or parenteral corticosteroid medications or immunosuppressants for a chronic medical condition. We investigated the rate of anemia (hematocrit $<35 \%$ ), thrombocytopenia $(<150 \times 103 / \mu \mathrm{L})$, and elevated serum creatinine (creatinine $>1 \mathrm{mg} / \mathrm{dL}$ ).

We performed 3 comparisons: (1) OSP versus AEEP, (2) OSP versus RASP, and (3) RASP versus AEEP. First, using a backward conditional logistic regression, we compared the 30-day perioperative procedural outcomes factored by organ system, prolonged operative time, and extended length of hospital stay (LOS $>1$ day) of the 3 procedures (RASP, AEEP, and OSP). Second, to mitigate the effect of procedure selection bias and potential confounding variables, propensity score matching (PSM) with replacement (giving priority to exact matches) was used to assess procedure-specific outcomes. This method would further decrease bias especially when dealing with very different numbers between the 3 arms[18]. Baseline demographics before and after matching are found in Supplementary Table 1. We defined major adverse cardiac event (MACE) as the incidence of one or more of the following: myocardial infarction, stroke/ cardiovascular events, cardiac arrest necessitating cardiopulmonary resuscitation (CPR), and death[19]. The pulmonary complications captured pneumonia and unplanned intubation events. We also identified patients with acute or progressive postoperative renal insufficiency requiring dialysis. Then, we gathered the occurrences of urinary tract infections, sepsis, and septic shock under infectious outcomes, and pulmonary embolism and deep venous thrombosis under venous thromboembolism (VTE). We also explored the rate of intraoperative and postoperative bleeding within the first 72 hours of surgery starting time. Moreover, we designated composite morbidity as the combination of pulmonary, renal, infectious, MACE, VTE, and transfusion outcomes. We further categorized ACS-National Surgical Quality Improvement Program (NSQIP) surgical outcomes in Clavien-Dindo grades according to previously published studies[20,21]. Clavien-Dindo grade I and grade II represent occurrences of surgical site infection (SSI), deep incisional SSI, organ space SSI, fascial dehiscence, pneumonia, urinary tract infections, deep venous thrombosis, progressive renal insufficiency, bleeding requiring transfusion. Grade III represents reoperation. Grade IV encompasses sepsis, septic shock, pulmonary embolism, myocardial infarction, cardiac arrest necessitating CPR, unplanned intubation, ventilator dependence $>48$ hours, acute renal failure necessitating dialysis, stroke, and coma. Grade V represents death.

\section{Statistics}

We compared patient demographics, comorbidities, and preoperative laboratory markers, and computed outcomes between the 3 procedures using MannWhitney $U$ test for continuous variables and chisquare test or Fisher exact test for categorical variables. Furthermore, a multivariate conditional backward logistic regression was constructed for each of the designated outcomes and adjusted for demographics, comorbidities, surgical procedure and the year the procedure was performed in.

Since observational studies are subject to treatmentselection bias, PSM allows estimation of treatment effect based on treatment assignment conditional to the accounted covariates[18,22]. Propensity score matching was used to adjust for covariates that could influence the choice of treatment of BPO: age, ASA class, body mass index, COPD, congestive heart failure, functional status, diabetes, hypertension, predisposition for bleeding, and the year of surgery to account for possible effect of the learning curve and changes in technique across the years. Using the generated propensity scores, weighted logistic regression analysis was conducted for the same outcomes. All analyses were conducted using the SPSS Statistics for Windows, version 25 (IBM Corp., Armonk, NY, US). 


\section{Results}

This series included 2867 AEEP, 234 RASP, and 1492 OSP procedures. Figure 2 depicts the annual trends for the different procedures. While RASP has a constant proportion over the years, OSP caseload decreased at the expense of more AEEP procedures. A total of $24.3 \%$, $25.2 \%$, and $27.3 \%$ of patients undergoing AEEP, RASP, and OSP, respectively, had an average age $>75$. Patient demographics and comorbidities were similar for all 3 procedures, except a higher proportion of patients undergoing AEEP had disseminated cancer (0.7\%), were on chronic steroid treatment $(2.5 \%)$, or were on anticoagulation (3.1\%); $(P=0.019, P=0.015, P=0.012$, respectively) (Table 1). There was also no difference in the rates of diabetes, hypertension, and COPD ( $P=0.967, P=0.470$, and $P=0.304$, respectively). In our cohort, there was an equal proportion of patients $(0.4 \%)$ on dialysis who underwent either of AEEP or OSP $(P>$ $0.05)$. Additionally, the AEEP group had the highest proportion of patients with hematocrit $<35 \%$ (22.9\%) and platelet count $<150 \mathrm{~K}(26.3 \%)$ while the OSP group had the highest proportion of patients with INR $\geq 1.1$ (19.1\%) $(P<0.001$ for all variables).

AEEP had the shortest median (interquartile range) operative time of the 3 procedures: 93 (59 to 131) minutes for AEEP versus 184 (149 to 234) minutes for RASP and 109 (82 to 152) minutes for OSP $(P<0.001$; Table 2). At the univariate level, fewer complications were observed with AEEP and RASP compared to OSP, yet there was no significant difference in rates of return to the operating room and 30-day mortality among the 3 procedures $(P>0.05)$.

After adjusting for other covariates, AEEP had decreased odds of postoperative pulmonary, renal, infectious, cardiac, and thromboembolic complications in comparison with OSP $(P<0.05)$ (Table 3$)$. AEEP versus RASP conferred lower odds of MACE $(\mathrm{OR}=0.18$; 95\% CI 0.04 to 0.75$)$ and VTE (OR $=0.22$; $95 \%$ CI 0.06 to 0.82 ). These findings were echoed in the PSM model. Furthermore, after matching, AEEP had lower odds of Clavien-Dindo grade I \& II adverse events (AEEP versus $\mathrm{OSP}, \mathrm{OR}=0.14 ; 95 \% \mathrm{CI} 0.10$ to 0.20 , and $\mathrm{AEEP}$ versus RASP, $\mathrm{OR}=0.40 ; 95 \%$ CI 0.26 to 0.62 ) (Table 4 ). AEEP also had reduced odds of grade IV complications (AEEP versus RASP, OR $=0.20$; 95\% CI 0.09 to 0.45 , and AEEP versus OSP, OR $=0.29 ; 95 \%$ CI 0.12 to 0.71 ) in comparison with OSP and RASP, respectively. Nevertheless, the type of surgery did not predict early postoperative mortality (Clavien-Dindo grade V). Despite having the largest proportion of patients with bleeding diathesis (3.1\%), AEEP was associated with a significantly lower risk for bleeding necessitating blood product transfusion than both OSP and AEEP; (AEEP versus OSP, OR $=0.06$; $95 \%$ CI 0.04 to 0.11 , and

\section{FIGURE 2.}

Annual trends for endoscopic enucleation of the prostate (AEEP) or robotic-assisted simple prostatectomy (RASP) or open simple prostatectomy (OSP)

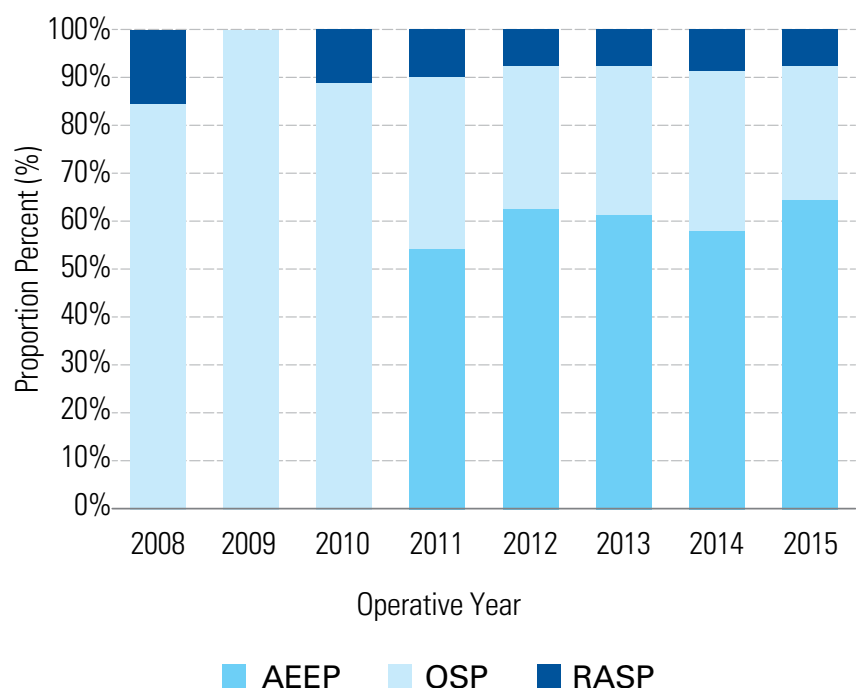

AEEP versus $\mathrm{RASP}, \mathrm{OR}=0.18 ; 95 \%$ CI 0.10 to 0.35 ). Additionally, AEEP was associated with a shorter LOS and decreased risk of overall morbidity than both OSP and RASP $(P<0.05)$. Similarly, the odds of operative time exceeding 90 minutes were lower for AEEP (AEEP versus $\mathrm{OSP}, \mathrm{OR}=0.56 ; 95 \% \mathrm{CI} 0.45$ to 0.70 ; $\mathrm{AEEP}$ versus RASP OR $=0.08 ; 95 \%$ CI 0.05 to 0.14 ).

\section{Discussion}

We used a real-world national database to provide objective operative outcome comparison between AEEP, RASP, and OSP: 3 procedures dedicated for the surgical management of symptomatic large prostates. When taking OSP as a reference, both AEEP and RASP had a shorter hospital stay and lower transfusion rates. Additionally, in comparison with OSP and RASP, AEEP had shorter operative time, shorter LOS, lower transfusion rates, and decreased odds of grade I and II as well as grade IV complications.

BPO resulting from large adenomas poses a more challenging decision-making process. The American Urological Association 2020 guidelines indicate that treatment options for large glands include open, laparoscopic, or robotically assisted simple prostatectomy, and that laser enucleation with RASP and AEEP have a lower risk profile because of their minimally invasive nature[4]. Meyer et al. documented the shift in numbers from OSP to RASP in the last decade, which they speculate occurred because the availability of robotics has made this the most costeffective approach and it requires no further training [23]. 


\section{TABLE 1.}

Baseline demographics, comorbidities, and laboratory markers among patients who have benign prostate obstruction who underwent anatomic endoscopic enucleation of the prostate (AEEP) or robotic assisted simple prostatectomy (RASP) or open simple prostatectomy (OSP)

\begin{tabular}{|c|c|c|c|c|}
\hline Variable & $\begin{array}{c}\text { AEEP }(N=2867) \\
n(\%)\end{array}$ & $\begin{array}{c}\text { RASP }(\mathbb{N}=234) \\
n(\%)\end{array}$ & $\begin{array}{c}\text { OSP (N =1492) } \\
n(\%)\end{array}$ & P-value \\
\hline Non-White & $259(9.7)$ & $32(14.2)$ & $210(15.4)$ & $<0.0001$ \\
\hline$\leq 65$ & 962 (33.9) & $66(28.2)$ & $407(27.4)$ & $<0.0001$ \\
\hline $65-\leq 75$ & 1187 (41.8) & $109(46.6)$ & $674(45.3)$ & \\
\hline$>75$ & $692(24.3)$ & $59(25.2)$ & 406 (27.3) & \\
\hline$A S A \geq 3$ & $1331(46.4)$ & $101(43.2)$ & $706(47.3)$ & 0.485 \\
\hline $\mathrm{BMI} \geq 30$ & $986(34.4)$ & $72(30.8)$ & $546(36.6)$ & 0.137 \\
\hline Diabetes & $522(18.2)$ & 44 (18.8) & $270(18.1)$ & 0.967 \\
\hline Smoker & $265(9.2)$ & $20(8.5)$ & $130(8.7)$ & 0.816 \\
\hline Dependent ${ }^{\mathrm{a}}$ & $52(1.8)$ & $2(0.9)$ & $20(1.3)$ & 0.396 \\
\hline COPD & $115(4.0)$ & $7(3.0)$ & $47(3.2)$ & 0.304 \\
\hline Heart failure & $12(0.4)$ & $0(0.0)$ & $4(0.3)$ & 0.747 \\
\hline Hypertension & $1,646(57.4)$ & $144(61.5)$ & $861(57.7)$ & 0.470 \\
\hline Dialysis & $11(0.4)$ & $0(0.0)$ & $6(0.4)$ & 1.0000 \\
\hline Disseminated cancer & $20(0.7)$ & $0(0)$ & $2(0.1)$ & 0.019 \\
\hline Chronic steroid & $73(2.5)$ & $4(1.7)$ & $19(1.3)$ & 0.015 \\
\hline Myocardial infarction & $2(0.1)$ & $0(0.0)$ & $0(0.0)$ & 0.603 \\
\hline Bleeding diathesis ${ }^{b}$ & $89(3.1)$ & $2(0.9)$ & $28(1.9)$ & 0.012 \\
\hline Creatinine $>1$ & $1,235(43.1)$ & 105 (44.9) & $686(46.0)$ & 0.182 \\
\hline Albumin $<3.5$ & $744(26.0)$ & $91(38.9)$ & $537(36.0)$ & $<0.0001$ \\
\hline Leukocyte $>6.5 \mathrm{~K}$ & $1,314(45.8)$ & $125(53.4)$ & $858(57.5)$ & $<0.0001$ \\
\hline Hematocrit <35\% & $656(22.9)$ & $29(12.4)$ & $249(16.7)$ & $<0.0001$ \\
\hline Platelet $<150 \mathrm{~K}$ & $753(26.3)$ & 44 (18.8) & $196(13.1)$ & $<0.0001$ \\
\hline INR $\geq 1.1$ & $366(12.8)$ & $31(13.2)$ & $285(19.1)$ & $<0.0001$ \\
\hline
\end{tabular}

ASA: American Society of Anesthesiology; BMI: body mass index; COPD: chronic obstructive pulmonary disorder;

INR: international normalized ratio.

a Dependent patients require partial or total assistance from another person for daily activities.

${ }^{b}$ Bleeding diathesis is defined as any chronic or persistent condition that places the patient at risk of excessive bleeding, including chronic anticoagulation that has not been discontinued prior to the procedure. 


\section{TABLE 2.}

Univariate analysis for postoperative outcomes following anatomic endoscopic enucleation of the prostate (AEEP), robotic-assisted simple prostatectomy (RASP), and open simple prostatectomy (OSP) for benign prostatic obstruction

\begin{tabular}{|c|c|c|c|c|}
\hline Variable & $\begin{array}{c}\text { AEEP } \\
(N=2867) \\
n(\%)\end{array}$ & $\begin{array}{c}\text { RASP } \\
(\mathbf{N}=234) \\
n(\%)\end{array}$ & $\begin{array}{c}\text { OSP } \\
(N=1492) \\
n(\%)\end{array}$ & P-value \\
\hline Pulmonarya & $8(0.3)$ & $2(0.9)$ & $26(1.7)$ & $<0.0001$ \\
\hline Renal $^{b}$ & $6(0.2)$ & $0(0.0)$ & $14(0.9)$ & 0.003 \\
\hline Infectiousc & $93(3.2)$ & $12(5.1)$ & $98(6.6)$ & $<0.0001$ \\
\hline MACE $^{d}$ & $8(0.3)$ & $3(1.3)$ & $15(1.0)$ & 0.002 \\
\hline VTEe & $8(0.3)$ & $3(1.3)$ & $29(1.9)$ & $<0.0001$ \\
\hline Transfusion ${ }^{f}$ & $38(1.3)$ & $16(6.8)$ & $275(18.4)$ & $<0.0001$ \\
\hline LOS $>1$ day & $441(15.4)$ & $157(67.1)$ & $1338(89.7)$ & $<0.0001$ \\
\hline Operative time & $93(59-131)$ & $184(149-234)$ & $109(82-152)$ & $<0.0001$ \\
\hline Return to OR & $54(1.9)$ & $6(2.6)$ & $45(3.0)$ & 0.057 \\
\hline mortality & $3(1.3)$ & $1(0.4)$ & $3(0.2)$ & 0.227 \\
\hline Composite morbidityg & $150(5.2)$ & $32(13.7)$ & $389(26.1)$ & $<0.0001$ \\
\hline
\end{tabular}

MACE: major adverse cardiac event; VTE: venous thromboembolism; LOS: length of hospital stay; OR: operating room.

a pneumonia and unplanned intubation; ${ }^{b}$ acute and progressive renal failure; ${ }^{c}$ urinary infections, sepsis, and septic shock; ${ }^{d}$ myocardial infarction, stroke/ cardiovascular events, cardiac arrest necessitating cardiopulmonary resuscitation, and death; ${ }^{e}$ pulmonary embolism and deep venous thrombosis; fbleeding requiring transfusion; gpulmonary, renal, infectious, MACE, VTE, transfusion outcomes.

Our results reflect the findings of a meta-analysis compiling 37 observational studies that explored the role of minimally invasive technique in simple prostatectomy and revealed that this procedure offers decreased blood loss and shorter LOS with comparable functional outcomes to OSP [24]. The largest reported outcome series for laparoscopic simple prostatectomy and RASP $(\mathrm{n}=1330)$ was from an American-European multicenter study[25]. The cohort had a median operative time of 100 minutes, a median estimated blood loss of $200 \mathrm{~mL}$, and a median LOS of 4 days[25]. Besides, $10.6 \%$ of the patients had a Clavien-Dindo grade l or grade 11 complication[25].
Although the findings are consistent with our results, no large studies have compared RASP with AEEP.

The patients in our cohort undergoing either procedure were similar in baseline characteristics with patient $\geq 75$ representing around a quarter of patients undergoing any of the 3 procedures[26]. On the other hand, patients on chronic anticoagulation were more likely to undergo AEEP. Although the risk of bleeding was considerable for patients on anticoagulation, the rate of transfusion was significantly lower with AEEP than with either OSP or RASP. This could be 


\section{TABLE 3.}

Multivariate backward conditional logistic regression comparing the perioperative outcomes of endoscopic anatomic enucleation of the prostate (AEEP), robotic-assisted simple prostatectomy (RASP), and open simple prostatectomy (OSP)

\begin{tabular}{|c|c|c|c|c|c|}
\hline $\begin{array}{l}\text { Postoperative } \\
\text { Outcomes }\end{array}$ & OSP & $\begin{array}{c}\text { AEEP } \\
\text { OR }(95 \% \text { CI })\end{array}$ & $\begin{array}{c}\text { RASP } \\
\text { OR (95\%CI) }\end{array}$ & RASP & $\begin{array}{c}\text { AEEP } \\
\text { OR }(95 \% \text { CI })\end{array}$ \\
\hline Pulmonary ${ }^{a}$ & Reference & $\begin{array}{c}0.19(0.09-0.44) \\
P<0.001\end{array}$ & $\begin{array}{c}0.62(0.14-2.68) \\
P=0.52\end{array}$ & Reference & - \\
\hline Renal $^{b}$ & Reference & $\begin{array}{c}0.14(0.04-0.47) \\
P=0.002\end{array}$ & - & Reference & - \\
\hline Infectious ${ }^{c}$ & Reference & $\begin{array}{c}0.49(0.36-0.66) \\
P<0.001\end{array}$ & $\begin{array}{c}0.89(0.48-1.66) \\
P=0.71\end{array}$ & Reference & $\begin{array}{c}0.54(0.29-1.01) \\
P=0.05\end{array}$ \\
\hline MACEd & Reference & $\begin{array}{c}0.27(0.11-0.67) \\
P=0.005\end{array}$ & $\begin{array}{c}1.12(0.30-4.19) \\
P=0.87\end{array}$ & Reference & $\begin{array}{c}0.18(0.04-0.75) \\
P=0.02\end{array}$ \\
\hline VTEe & Reference & $\begin{array}{c}0.16(0.07-0.35) \\
P<0.001\end{array}$ & $\begin{array}{c}0.59(0.17-2.03) \\
P=0.40\end{array}$ & Reference & $\begin{array}{c}0.22(0.06-0.82) \\
P=0.03\end{array}$ \\
\hline OT $>90 \mathrm{~min}$ & Reference & $\begin{array}{c}0.48(0.42-0.56) \\
P<0.001\end{array}$ & $\begin{array}{c}7.36(4.32-12.53) \\
P<0.001\end{array}$ & Reference & $\begin{array}{c}0.09(0.05-0.15) \\
P<0.001\end{array}$ \\
\hline Transfusion ${ }^{f}$ & Reference & $\begin{array}{c}0.04(0.03-0.07) \\
P<0.001\end{array}$ & $\begin{array}{c}0.30(0.17-0.52) \\
P<0.001\end{array}$ & Reference & $\begin{array}{c}0.14(0.07-0.27) \\
P<0.001\end{array}$ \\
\hline LOS $>1$ day & Reference & $\begin{array}{c}0.02(0.01-0.03) \\
P<0.001\end{array}$ & $\begin{array}{c}0.18(0.13-0.27) \\
P<0.001\end{array}$ & Reference & $\begin{array}{c}0.08(0.06-0.12) \\
P<0.001\end{array}$ \\
\hline $\begin{array}{l}\text { Composite } \\
\text { morbidityg }\end{array}$ & Reference & $\begin{array}{c}0.14(0.11-0.18) \\
P<0.001\end{array}$ & $\begin{array}{c}0.45(0.30-0.68) \\
P<0.001\end{array}$ & Reference & $\begin{array}{c}0.30(0.20-0.46) \\
P<0.001\end{array}$ \\
\hline \multicolumn{6}{|c|}{ Clavien-Dindo Grade Complications } \\
\hline Grade I \& || CD & Reference & $\begin{array}{c}0.12(0.10-0.16) \\
P<0.001\end{array}$ & $\begin{array}{c}0.38(0.24-9.58) \\
P<0.001\end{array}$ & Reference & $\begin{array}{c}0.30(0.19-0.48) \\
P<0.001\end{array}$ \\
\hline Return to OR & Reference & - & - & Reference & - \\
\hline Grade IV CD & Reference & $\begin{array}{c}0.26(0.16-0.42) \\
P<0.001\end{array}$ & - & Reference & $\begin{array}{c}0.05(0.01-0.24) \\
P<0.001\end{array}$ \\
\hline Mortality & Reference & - & - & Reference & - \\
\hline
\end{tabular}

CD: Clavien-Dindo; Cl: confidence interval MACE: major adverse cardiac event; VTE: venous thromboembolism; OR: odds ratio; OT: operative time a pneumonia and unplanned intubation; ${ }^{b}$ acute and progressive renal failure; ${ }^{c}$ urinary infections, sepsis, and septic shock; ${ }^{d}$ myocardial infarction, stroke/ cardiovascular events, cardiac arrest necessitating cardiopulmonary resuscitation, and death, ${ }^{e}$ pulmonary embolism and deep venous thrombosis; ${ }^{f}$ bleeding requiring transfusion; ${ }^{9}$ pulmonary, renal, infectious, MACE, VTE, transfusion outcomes. Logistic regression model adjusted for race, age, American Society of Anesthesiology (ASA) class, functional dependence, body mass index (BMI), smoking, diabetes, hypertension, predisposition to bleeding (includes anticoagulation), chronic obstructive pulmonary disease (COPD), history of heart failure, dialysis dependence, chronic steroid use, history of disseminated cancer, laboratory markers (creatinine, leukocyte count, hematocrit, platelet count), and surgical procedure (AEEP versus RASP versus OSP), and year of operation. 


\section{TABLE 4.}

Propensity score matching comparing the perioperative outcomes of anatomic endoscopic enucleation of the prostate (AEEP), robotic-assisted simple prostatectomy (RASP), and open simple prostatectomy (OSP)

\begin{tabular}{|c|c|c|c|c|c|}
\hline $\begin{array}{l}\text { Postoperative } \\
\text { Outcomes }\end{array}$ & OSP & $\begin{array}{c}\text { AEEP } \\
\text { OR }(95 \% \text { CI) }\end{array}$ & $\begin{array}{c}\text { RASP } \\
\text { OR }(95 \% \text { CI) }\end{array}$ & RASP & $\begin{array}{c}\text { AEEP } \\
\text { OR }(95 \% \text { CI })\end{array}$ \\
\hline Pulmonarya & Reference & $\begin{array}{c}0.15(0.04-0.60) \\
P=0.007\end{array}$ & $\begin{array}{c}0.45(0.09-2.28) \\
P=0.336\end{array}$ & Reference & $\begin{array}{c}0.36(0.07-1.92) \\
P=0.228\end{array}$ \\
\hline Renal $^{b}$ & Reference & $\begin{array}{c}0.12(0.02-0.90) \\
P=0.04\end{array}$ & - & Reference & - \\
\hline Infectious ${ }^{c}$ & Reference & $\begin{array}{c}0.47(0.28-0.77) \\
P=0.003\end{array}$ & $\begin{array}{c}0.79(0.41-1.54) \\
P=0.493\end{array}$ & Reference & $\begin{array}{c}0.61(0.32-1.17) \\
P=0.608\end{array}$ \\
\hline MACE $^{d}$ & Reference & $\begin{array}{c}0.26(0.06-1.15) \\
P=0.08\end{array}$ & $\begin{array}{c}1.32(0.35-5.00) \\
P=0.681\end{array}$ & Reference & $\begin{array}{c}0.22(0.05-0.87) \\
P=0.03\end{array}$ \\
\hline VTEe & Reference & $\begin{array}{c}0.13(0.03-0.50) \\
P=0.003\end{array}$ & $\begin{array}{c}0.67(0.18-2.44) \\
P=0.544\end{array}$ & Reference & $\begin{array}{c}0.26(0.09-0.92) \\
P=0.03\end{array}$ \\
\hline $\mathrm{OT}>90 \mathrm{~min}$ & Reference & $\begin{array}{c}0.56(0.45-0.70) \\
P<0.001\end{array}$ & $\begin{array}{c}6.01(3.45-10.45) \\
P<0.001\end{array}$ & Reference & $\begin{array}{c}0.08(0.05-0.14) \\
P<0.001\end{array}$ \\
\hline Transfusion ${ }^{f}$ & Reference & $\begin{array}{c}0.06(0.04-0.11) \\
P<0.001\end{array}$ & $\begin{array}{c}0.33(0.19-0.59) \\
P<0.001\end{array}$ & Reference & $\begin{array}{c}0.18(0.10-0.35) \\
P<0.001\end{array}$ \\
\hline LOS $>1$ day & Reference & $\begin{array}{c}0.02(0.01-0.03) \\
P<0.001\end{array}$ & $\begin{array}{c}0.24(0.17-0.35) \\
P<0.001\end{array}$ & Reference & $\begin{array}{c}0.09(0.06-0.12) \\
P<0.001\end{array}$ \\
\hline $\begin{array}{l}\text { Composite } \\
\text { morbidityg }\end{array}$ & Reference & $\begin{array}{c}0.15(0.11-0.22) \\
P<0.001\end{array}$ & $\begin{array}{c}0.46(0.30-0.71) \\
P<0.001\end{array}$ & Reference & $\begin{array}{c}0.35(0.22-0.53) \\
P<0.001\end{array}$ \\
\hline \multicolumn{6}{|c|}{ Clavien-Dindo Grade Complications } \\
\hline Grade I \& II & Reference & $\begin{array}{c}0.14(0.10-0.20) \\
P<0.001\end{array}$ & $\begin{array}{c}0.40(0.26-0.62) \\
P<0.001\end{array}$ & Reference & $\begin{array}{c}0.36(0.23-0.56) \\
P<0.001\end{array}$ \\
\hline Return to OR & Reference & - & - & Reference & - \\
\hline Grade IV & Reference & $\begin{array}{c}0.20(0.09-0.45) \\
P<0.001\end{array}$ & $\begin{array}{c}0.76(0.32-1.79) \\
P=0.527\end{array}$ & Reference & $\begin{array}{c}0.29(0.12-0.71) \\
P=0.007\end{array}$ \\
\hline Mortality & Reference & $\begin{array}{c}0.56(0.03-9.86) \\
P=0.693\end{array}$ & $\begin{array}{c}2.19(0.20-23.36) \\
P=0.518\end{array}$ & Reference & $\begin{array}{c}0.23(0.02-2.39) \\
P=0.220\end{array}$ \\
\hline
\end{tabular}

CD: Clavien-Dindo; Cl: confidence interval; MACE: major adverse cardiac event; VTE: venous thromboembolism; OR: odds ratio; OT: operative time. Covariates included in the PSM model were age category, ASA class, BMI, COPD, congestive heart failure, hypertension, diabetes, functional status, predisposition to bleeding, and year of operation. 
attributed to the use of lasers, with the ability to heat well-vascularized tissue thereby counteracting bleeding[27]. Further, a high power (80 to $100 \mathrm{~W}$ ) energy source can achieve simultaneous cutting and coagulation[27]. Consequently, AEEP is a safer modality than RASP for the treatment of patients on anticoagulation $[28,29]$. Furthermore, our results echo the findings of other studies that show AEEP is associated with the least blood loss among the 3 modalities $[8,30]$.

In our study, AEEP is also associated with a shorter operative time and shorter hospital stay. These findings are in concordance with the literature where a prolonged operative time is attributable to technical factors of robotic surgery, such as applying the ports and docking the robot[11-13]. In our study, the median operative time for RASP is slightly longer (184 [149 to 234 minutes) than reported in the literature (150 to 160 minutes). A systematic review by Kordan et al. also revealed a shorter average operative time for OSP (79 to 93 minutes) depending on the surgical series[15]. This is probably because the ACS NSQIP database reflects realworld outcomes of different levels of surgical expertise $[23,25]$. No information is available on catheterization time, but if we assume that the LOS is a surrogate for catheterization time, AEEP should have a shorter catheterization time $[12,13,25]$. An increased need for continuous bladder irrigation because of bleeding may also lengthen hospital stay after RASP and OSP compared with AEEP.

RASP is estimated to be more costly than its open counterpart and costs twice as much as AEEP[31]. The case has been made that the procurement of AEEP to hospitals is costly and necessitates a steeper learning curve[23]. However, diverse endonucleation techniques have an acceptable learning curve of 25 to 50 cases with similar perioperative and functional outcomes[32,33]. Therefore, the availability of the modality and the surgeon's level of comfort would dictate the type of surgery offered to patients. Hence, AEEP and RASP have both mitigated the risks associated with OSP while conserving equivalent functional outcomes; however, AEEP offers the advantage of a shorter operative time, reduced LOS, and safer profile than RASP [34]. In comparison to transurethral resection of the prostate, AEEP has also demonstrated superiority as it reduces the risk of postoperative urinary tract infections and decreases the LOS[35]. Therefore, AEEP could be preferable for large adenomas except in the presence of co-existing conditions such as large bladder calculi that would require a long operative time to eradicate endoscopically, or diverticula where the robotic technique would more efficiently manage both conditions concomitantly[23,36].

Treatment cost encompasses surgical instruments, complications, and hospital stay among others. In comparison to OSP, RASP has higher operating expenses (USD 2797), but its safer profile (decreased transfusion rate and shorter LOS) might render it more cost-effective than its open counterpart[15,37]. On the other hand, AEEP (HoLEP) was found to be 9.6\% (EUR 2356) cheaper than OSP[38]. The surgeons' expertise and the institution caseload directly impact the perioperative and functional outcomes, which in turn influence costs. Costs also vary according to country, type of institution (public versus private, military versus civil), and type of cost analysis conducted. Further studies with more precise comparisons are needed to assess the costs of the 3 procedures[15].

Our study is limited by its retrospective nature. While national databases like NSQIP allow outcome comparison among a large sample population, they might not include surgery-specific clinical data such as prostate size, average gram resected per minute, and functional outcomes such as post-void residual, International Prostate Symptom Score, quality of life scores, and incontinence rates. Additionally, large databases do not consider surgeon experience and learning curve on the outcome. Another limitation inherent to the database is the inability to capture events occurring more than 30 days after the procedure. Moreover, we were not able to assess continence, lower urinary tract symptoms, and sexual function because they are not captured in NSQIP. Furthermore, we were unable to control for the selection bias that results from the choice of procedure being highly dependent on the expertise available in each institution. However, we conducted 2 forms of analysis to cross-check our results. Our methodology might not have captured all RASP procedures performed in the study period. Nonetheless, this study provides an accurate assessment of the feasibility of the use of AEEP and RASP by a large number of surgeons with a wide range of expertise and experience.

\section{Conclusion}

We report the outcomes of OSP, AEEP, and RASP in a large real-world cohort of patients. AEEP was found to have a safer profile than OSP and RASP as it conferred a shorter operative time, shorter hospital stay, and lower incidence of postoperative complications. Thus, AEEP could be advantageous for frail patients with large prostates or for those dependent on chronic anticoagulation. 


\section{Author Contributions}

Muhieddine Labban: Methodology, formal analysis, writing-original draft

Nassib Abou Heidar: Conceptualization, methodology, writing-original draft

Vincent Misrai: Validation, writing — review and editing

Jad Najdi: Writing-original draft

Hani Tamim: Data curation, project administration

Albert El-Hajj: Conceptualization, methodology, writing-review and editing, supervision

All authors read and approved the final version of the manuscript.

\section{References}

1. Egan KB. The Epidemiology of benign prostatic hyperplasia associated with lower urinary tract symptoms: prevalence and incident rates. Urol Clin North Am.2016;43(3):289-97. Epub 2016/08/01. doi: 10.1016/j. ucl.2016.04.001. PubMed PMID: 27476122.

2. Wei JT, Calhoun E, Jacobsen SJ. Urologic diseases in America project: benign prostatic hyperplasia. J Urol.2008;179(5 Suppl):S75-80. Epub 2008/04/25. doi: 10.1016/j.juro.2008.03.141. PubMed PMID: 18405761.

3. Gratzke C, Bachmann A, Descazeaud A, Drake MJ, Madersbacher $S$, Mamoulakis $C$, et al. EAU guidelines on the assessment of non-neurogenic male lower urinary tract symptoms including benign prostatic obstruction. Eur Urol.2015;67(6):1099-109. Epub 2015/01/24. doi: 10.1016/j.eururo.2014.12.038. PubMed PMID: 25613154

4. AUA Announces Updates to Clinical Guidance for Surgical Management of LUTS Attributed to BPH. 2020. Epub June 25.2020.

5. Stolzenburg JU, Kallidonis P, Qazi H, Ho Thi P, Dietel A, Liatsikos EN, et al. Extraperitoneal approach for robotic-assisted simple prostatectomy. Urology.2014;84(5):1099-105. Epub 2014/12/03. doi: 10.1016/j.urology.2014.06.045. PubMed PMID: 25443912.

6. Shah AA, Gahan JC, Sorokin I. Comparison of robot-assisted versus open simple prostatectomy for benign prostatic hyperplasia. Curr Urol Rep.2018;19(9):71-. doi: 10.1007/s11934-018-0820-1. PubMed PMID: 29998354.

7. Mourmouris P, Keskin SM, Skolarikos A, Argun OB, Karagiannis AA, Tufek I, et al. A prospective comparative analysis of robot-assisted vs open simple prostatectomy for benign prostatic hyperplasia. BJU Int.2019;123(2):313-7. Epub 10/15. doi: 10.1111/bju.14531. PubMed PMID: 30198196.

8. Lin $Y$, Wu X, Xu A, Ren R, Zhou X, Wen Y, et al. Transurethral enucleation of the prostate versus transvesical open prostatectomy for large benign prostatic hyperplasia: a systematic review and metaanalysis of randomized controlled trials. World J Urol.2016;34(9):120719. Epub 2015/12/25. doi: 10.1007/s00345-015-1735-9. PubMed PMID: 26699627.

\section{Acknowledgements}

The ACS NSQIP and the hospitals participating in the ACS NSQIP are the source of the data used herein; they have not verified and are not responsible for the statistical validity of the data analysis or the conclusions derived by the authors.

9. Gilling PJ, Cass CB, Cresswell MD, Fraundorfer MR. Holmium laser resection of the prostate: preliminary results of a new method for the treatment of benign prostatic hyperplasia. Urology.1996;47(1):48-51. Epub 1996/01/01. doi: 10.1016/s0090-4295(99)80381-1. PubMed PMID: 8560662

10. Gilling PJ, Wilson LC, King CJ, Westenberg AM, Frampton CM, Fraundorfer MR. Long-term results of a randomized trial comparing holmium laser enucleation of the prostate and transurethral resection of the prostate: results at 7 years. BJU Int.2012;109(3):408-11. Epub 2011/09/03. doi: 10.1111/j.1464-410X.2011.10359.x. PubMed PMID: 21883820

11. Zhang MW, El Tayeb MM, Borofsky MS, Dauw CA, Wagner KR, Lowry PS, et al. Comparison of perioperative outcomes between holmium laser enucleation of the prostate and robot-assisted simple prostatectomy. J Endourol.2017;31(9):847-50. Epub 2017/06/24. doi: 10.1089/end.2017.0095. PubMed PMID: 28637364.

12. Umari P, Fossati N, Gandaglia G, Pokorny M, De Groote R, Geurts $\mathrm{N}$, et al. Robotic assisted simple prostatectomy versus holmium laser enucleation of the prostate for lower urinary tract symptoms in patients with large volume prostate: a comparative analysis from a high volume center. J Urol.2017;197(4):1108-14. Epub 2016/09/13. doi: 10.1016/j.juro.2016.08.114. PubMed PMID: 27615435.

13. Baldini A, Fassi-Fehri H, Duarte RC, Crouzet S, Ecochard R, Abid N, et al. Holmium laser enucleation of the prostate versus laparoscopic transcapsular prostatectomy: perioperative results and threemonth follow-up. Curr Urol.2017;10(2):81-6. Epub 05/30. doi: 10.1159/000447156. PubMed PMID: 28785192.

14. Sorokin I, Sundaram V, Singla N, Walker J, Margulis V, Roehrborn $C$, et al. Robot-assisted versus open simple prostatectomy for benign prostatic hyperplasia in large glands: a propensity scorematched comparison of perioperative and short-term outcomes. J Endourol.2017;31(11):1164-9. Epub 2017/09/01. doi: 10.1089/ end.2017.0489. PubMed PMID: 28854815.

15. Kordan Y, Canda AE, Köseoğlu E, Balbay D, Laguna MP, de la Rosette J. Robotic-assisted simple prostatectomy: a systematic review. J Clin Med.2020;9(6). Epub 2020/06/13. doi: 10.3390/jcm9061798. PubMed PMID: 32527020; PubMed Central PMCID: PMCPMC7356910. 
16. Surgeons ACo. ACS NSQIP Hospitals 2020 [cited 2020 July 20]. Available from: https://www.facs.org/search/nsqip-participants?allresults=.

17. Lerner LB, Rajender A. Laser prostate enucleation techniques. Can J Urol.2015;22(Suppl 1):53-9. Epub 2015/10/27. PubMed PMID: 26497344.

18. StuartEA. Matching methods for causal inference: a review and a look forward. Stat Sci.2010;25(1):1-21. doi: 10.1214/09-STS313. PubMed PMID: 20871802.

19. Berger A, Simpson A, Bhagnani T, Leeper NJ, Murphy B, Nordstrom B, et al. Incidence and cost of major adverse cardiovascular events and major adverse limb events in patients with chronic coronary artery disease or peripheral artery disease. Am J Cardiol.2019;123(12):1893-9. Epub 03/16. doi: 10.1016/j.amjcard.2019.03.022. PubMed PMID: 31014542.

20. Dindo D, Demartines N, Clavien PA. Classification of surgical complications: a new proposal with evaluation in a cohort of 6336 patients and results of a survey. Ann Surg.2004;240(2):205-13. Epub 2004/07/27. doi: 10.1097/01.sla.0000133083.54934.ae. PubMed PMID: 15273542; PubMed Central PMCID: PMCPMC1360123.

21. Shahait M, Labban M, Dobbs RW, Cheaib JG, Lee DI, Tamim H, et al. A 5-Item frailty index for predicting morbidity and mortality after radical prostatectomy: an analysis of the American College of Surgeons national surgical quality improvement program database. J Endourol.2021. Epub 2020/09/17. doi: 10.1089/end.2020.0597. PubMed PMID: 32935596.

22. Austin PC, Stuart EA. Moving towards best practice when using inverse probability of treatment weighting (IPTW) using the propensity score to estimate causal treatment effects in observational studies. Stat Med.2015;34(28):3661-79. Epub 2015/08/03. doi: 10.1002/ sim.6607. PubMed PMID: 26238958.

23. Meyer D, Weprin S, Zukovski EB, Porpiglia F, Hampton LJ, Autorino R. Rationale for robotic-assisted simple prostatectomy for benign prostatic obstruction. Eur Urol Focus.2018;4(5):643-7. doi: https://doi. org/10.1016/j.euf.2018.07.007.

24. Lucca I, Shariat SF, Hofbauer SL, Klatte T. Outcomes of minimally invasive simple prostatectomy for benign prostatic hyperplasia: a systematic review and meta-analysis. World J Urol.2015;33(4):563-70. doi: 10.1007/s00345-014-1324-3.

25. Autorino R, Zargar H, Mariano MB, Sanchez-Salas R, Sotelo RJ, Chlosta PL, et al. Perioperative outcomes of robotic and laparoscopic simple prostatectomy: a European-American multi-institutional analysis. Eur Urol.2015;68(1):86-94. doi: https://doi.org/10.1016/j. eururo.2014.11.044.

26. Elshal AM, Elmansy HM, Elhilali MM. Transurethral laser surgery for benign prostate hyperplasia in octogenarians: safety and outcomes. Urology.2013;81(3):634-9. Epub 2013/01/22. doi: 10.1016/j. urology.2012.11.042. PubMed PMID: 23332997.

27. Sivarajan G, Borofsky MS, Shah 0, Lingeman JE, Lepor H. The role of minimally invasive surgical techniques in the management of large-gland benign prostatic hypertrophy. Rev Urol.2015;17(3):140-9. PubMed PMID: 26543428.
28. Rivera M, Krambeck A, Lingeman J. Holmium laser enucleation of the prostate in patients requiring anticoagulation. Curr Urol Rep.2017;18(10):77. Epub 2017/08/07. doi: 10.1007/s11934-017-0727-2. PubMed PMID: 28780634.

29. El Tayeb MM, Jacob JM, Bhojani N, Bammerlin E, Lingeman JE. holmium laser enucleation of the prostate in patients requiring anticoagulation. J Endourol.2016;30(7):805-9. Epub 05/03. doi: 10.1089/end.2016.0070. PubMed PMID: 27065437.

30. Li J, Cao D, Peng L, Ren Z, Gou H, Li Y, et al. comparison between minimally invasive simple prostatectomy and open simple prostatectomy for large prostates: a systematic review and metaanalysis of comparative trials. J Endourol.2019;33(9):767-76. Epub 2019/06/28. doi: 10.1089/end.2019.0306. PubMed PMID: 31244334.

31. Juaneda R, Thanigasalam R, Rizk J, PerrotE, Theveniaud PE, Baumert H. Holmium laser enucleation versus laparoscopic simple prostatectomy for large adenomas. Actas Urol Esp.2016;40(1):43-8. Epub 07/29. doi: 10.1016/j.acuro.2015.05.010. PubMed PMID: 26233479.

32. Kampantais S, Dimopoulos P, Tasleem A, Acher P, Gordon K, Young A. assessing the learning curve of holmium laser enucleation of prostate (HoLEP). A systematic review. Urology.2018;120:9-22. Epub 07/04. doi: 10.1016/j.urology.2018.06.012. PubMed PMID: 30403609

33. Peyronnet B, Robert G, Comat V, Rouprêt M, Gomez-Sancha F, Cornu J-N, et al. Learning curves and perioperative outcomes after endoscopic enucleation of the prostate: a comparison between GreenLight 532-nm and holmium lasers. World J Urol.2017;35(6):97383. Epub 10/20. doi: 10.1007/s00345-016-1957-5. PubMed PMID: 27766387.

34. Humphreys MR, Miller NL, Handa SE, Terry C, Munch LC, Lingeman JE. Holmium laser enucleation of the prostate--outcomes independent of prostate size? J Urol.2008;180(6):2431-5. Epub 10/19. doi: 10.1016/j. juro.2008.08.019. PubMed PMID: 18930490.

35. Heidar NA, Labban M, Misrai V, Mailhac A, Tamim H, El-Hajj A. Laser enucleation of the prostate versus transurethral resection of the prostate: perioperative outcomes from the ACS NSQIP database. World J Urol.2020. doi: 10.1007/s00345-020-03100-7.

36. Nestler S, Bach T, Herrmann T, Jutzi S, Roos FC, Hampel C, et al. Surgical treatment of large volume prostates: a matched pair analysis comparing the open, endoscopic (ThuVEP) and robotic approach. World J Urol.2019;37(9):1927-31. Epub 12/04. doi: 10.1007/s00345-0182585-z. PubMed PMID: 30515596.

37. Sutherland DE, Perez DS, Weeks DC. Robot-assisted simple prostatectomy for severe benign prostatic hyperplasia. J Endourol. 2011;25(4):641-4. Epub 2011/03/19. doi: 10.1089/end.2010.0528. PubMed PMID: 21413877.

38. Salonia A, Suardi N, Naspro R, Mazzoccoli B, Zanni G, Gallina $A$, et al. Holmium laser enucleation versus open prostatectomy for benign prostatic hyperplasia: an inpatient cost analysis. Urology.2006;68(2):302-6. Epub 2006/08/15. doi: 10.1016/j.urology. 2006.02.007. PubMed PMID: 16904441. 


\section{SUPPLEMENTARY TABLE $1 A$.}

Comparison of baseline demographics before and after propensity score matching for anatomic enucleation of the prostate (AEEP) and open simple prostatectomy (OSP)

\begin{tabular}{|c|c|c|c|c|c|c|}
\hline \multirow{3}{*}{ Variable } & \multicolumn{3}{|c|}{ Before matching } & \multicolumn{3}{|c|}{ After matching } \\
\hline & OSP & AEEP & \multirow{2}{*}{$P$-value } & OSP & AEEP & \multirow{2}{*}{$P$-value } \\
\hline & n (\%) & n (\%) & & $n(\%)$ & $n(\%)$ & \\
\hline Age $\geq 68^{*}$ (median age) & $861(57.9)$ & $1488(52.4)$ & 0.001 & $323(61.5)$ & $531(55.7)$ & 0.03 \\
\hline$A S A \geq 3$ & 706 (47.3) & $1331(46.4)$ & 0.587 & $252(48.1)$ & $443(46.4)$ & 0.549 \\
\hline Dependent functional status & $52(1.8)$ & $20(1.3)$ & 0.262 & $6(1.1)$ & $14(1.5)$ & 0.649 \\
\hline $\mathrm{BMI} \geq 30$ & $546(36.6)$ & $986(34.4)$ & 0.151 & $200(38.2)$ & $345(36.1)$ & 0.464 \\
\hline Diabetes mellitus & $270(18.1)$ & $522(18.2)$ & 0.934 & $96(18.3)$ & $174(18.2)$ & 0.962 \\
\hline COPD & $47(3.2)$ & $115(4.0)$ & 0.177 & $14(2.7)$ & $32(3.4)$ & 0.533 \\
\hline Congestive heart failure & $4(0.3)$ & $12(0.4)$ & 0.6 & $1(0.2)$ & $2(0.2)$ & 0.938 \\
\hline Hypertension & $861(57.7)$ & $1646(57.4)$ & 0.872 & $303(57.8)$ & $549(57.5)$ & 0.912 \\
\hline Bleeding disorder & $28(1.9)$ & $89(3.1)$ & 0.018 & $8(1.5)$ & $20(2.1)$ & 0.551 \\
\hline Year of operation $\geq 2014$ & $988(66.2)$ & $2072(72.3)$ & $<0.001$ & $315(60.0)$ & 642 (67.2) & 0.006 \\
\hline
\end{tabular}




\section{SUPPLEMENTARY TABLE 1B.}

Comparison of baseline demographics before and after propensity score matching for robotic-assisted simple prostatectomy (RASP) and open simple prostatectomy (OSP)

\begin{tabular}{|c|c|c|c|c|c|c|}
\hline \multirow{3}{*}{ Variable } & \multicolumn{3}{|c|}{ Before matching } & \multicolumn{3}{|c|}{ After matching } \\
\hline & OSP & RASP & \multirow{2}{*}{$P$-value } & OSP & RASP & \multirow{2}{*}{$P$-value } \\
\hline & n (\%) & n (\%) & & n (\%) & $n(\%)$ & \\
\hline Age $\geq 68$ (median age) & $861(57.9)$ & $133(56.8)$ & 0.759 & $741(57.8)$ & $113(57.4)$ & 0.938 \\
\hline$A S A \geq 3$ & $706(47.3)$ & $101(43.2)$ & 0.260 & $610(47.5)$ & $85(43.4)$ & 0.283 \\
\hline Dependent functional status & $20(1.3)$ & $2(0.9)$ & 0.758 & $18(1.4)$ & $2(1.0)$ & 0.761 \\
\hline $\mathrm{BMI} \geq 30$ & $546(36.6)$ & $72(30.8)$ & 0.092 & $483(37.6)$ & $62(31.6)$ & 0.112 \\
\hline Diabetes mellitus & $270(18.1)$ & $44(18.8)$ & 0.855 & $234(18.2)$ & $36(18.4)$ & 1 \\
\hline COPD & $47(3.2)$ & $7(3.0)$ & 1 & $40(3.1)$ & $6(3.0)$ & 1 \\
\hline Congestive heart failure & $4(0.3)$ & $0(0.0)$ & 0.649 & $3(0.2)$ & $0(0.0)$ & 1 \\
\hline Hypertension & $861(57.7)$ & $144(61.5)$ & 0.285 & 732 (57.1) & $120(60.9)$ & 0.316 \\
\hline Bleeding disorder & $28(1.9)$ & $2(0.9)$ & 0.305 & $26(2.0)$ & $2(1.0)$ & 0.416 \\
\hline Year of operation $\geq 2014$ & $988(66.2)$ & $120(51.3)$ & $<0.001$ & $883(68.9)$ & $105(53.6)$ & $<0.001$ \\
\hline
\end{tabular}




\section{SUPPLEMENTARY TABLE 1C.}

Comparison of baseline demographics before and after propensity score matching for open simple prostatectomy (OSP) and robotic assisted simple prostatectomy (RASP)

\begin{tabular}{|c|c|c|c|c|c|c|}
\hline \multirow{3}{*}{ Variable } & \multicolumn{3}{|c|}{ Before matching } & \multicolumn{3}{|c|}{ After matching } \\
\hline & RASP & AEEP & \multirow{2}{*}{$P$-value } & OSP & RASP & \multirow{2}{*}{ P-value } \\
\hline & n (\%) & $n(\%)$ & & $n(\%)$ & $n(\%)$ & \\
\hline Age $\geq 68$ (median age) & $133(56.8)$ & $1488(52.4)$ & 0.189 & $177(56.5)$ & $1357(51.9)$ & 0.219 \\
\hline$A S A \geq 3$ & $101(43.2)$ & $1331(46.4)$ & 0.341 & 88 (42.7) & $1211(46.3)$ & 0.345 \\
\hline Dependent functional status & $2(0.9)$ & $52(1.8)$ & 0.321 & $2(1.0)$ & $44(1.7)$ & 0.578 \\
\hline $\mathrm{BMI} \geq 30$ & 72 (30.8) & $986(34.4)$ & 0.282 & 64 (31.1) & $915(35.0)$ & 0.287 \\
\hline Diabetes mellitus & $101(43.2)$ & $1331(46.4)$ & 0.341 & $38(18.4)$ & $477(18.2)$ & 1 \\
\hline COPD & $2(0.9)$ & $52(1.8)$ & 0.321 & $6(2.9)$ & $106(4.1)$ & 0.468 \\
\hline Congestive heart failure & $72(30.8)$ & $986(34.4)$ & 0.282 & $0(0.0)$ & $12(0.5)$ & 0.619 \\
\hline Hypertension & $101(43.2)$ & $1331(46.4)$ & 0.341 & $126(60.9)$ & $1492(57.1)$ & 0.307 \\
\hline Bleeding disorder & $2(0.9)$ & $89(3.1)$ & 0.065 & $2(1.0)$ & $85(3.3)$ & 0.089 \\
\hline Year of operation $\geq 2014$ & $120(51.3)$ & 2072 (72.3) & $<0.001$ & $112(54.4)$ & $1946(74.4)$ & $<0.001$ \\
\hline
\end{tabular}

ASA: American Society of Anesthesia; BMI: body mass index; COPD: chronic obstructive pulmonary disease 